\title{
Simulation and Experimental Research on Overvoltage Measurement of Hybrid CVT
}

\author{
Yu Nie ${ }^{1}$, Guoqing Zhang ${ }^{1}$, Jiakuo Song ${ }^{1}$ and Guizhong Wang ${ }^{2}$ \\ ${ }^{1}$ Department of Electrical Engineering, Harbin Institute of Technology, Harbin, Heilongjiang, China \\ ${ }^{2}$ Hebei Key Laboratory of Grid Advanced Measurement and Monitor
}

\begin{abstract}
The CVT equipment running on site is slightly modified to form a hybrid CVT to achieve transient overvoltage measurement, which is theoretically feasible and practical. In order to study the voltage division method based on the hybrid CVT to measure the overvoltage of the power system, that is, the low voltage capacitor $\mathrm{C} 3$ is connected in series between the low voltage terminal of the medium voltage capacitor $\mathrm{C} 2$ of the traditional CVT and the ground, and the transient overvoltage measurement is realized through C3. This article uses ATP-EMTP to establish its high-frequency transient model for simulation analysis. For further verification, a power frequency voltage test and an impulse voltage test were carried out on the $110 \mathrm{kV}$ hybrid CVT produced by Taikai Transformer Factory, focusing on the analysis of the partial voltage characteristics of the capacitor $\mathrm{C} 3$. The simulation results show that the $\mathrm{C} 3$ partial pressure method can achieve a relatively accurate measurement of the transient overvoltage. The test results show that the voltage across $\mathrm{C} 3$ is affected by uncertain factors and has an impact phenomenon. The above analysis process and results have certain practical value for designing actual products.
\end{abstract}

\section{Introduction}

In the power system, capacitive voltage transformers (CVT) are mainly used for high-voltage voltage measurement equipment[1], but it focuses on power frequency signal measurement and cannot cope with the challenges posed by broadband signal measurement, and transient overvoltage signals contain Abundant frequencies, so there is currently a lack of complete transient overvoltage measurement devices. Due to the inertial components such as inductance and capacitance inside the capacitive voltage transformer, the measurement frequency range is narrow, and the measurement is accurate only under the power frequency condition. Moreover, its transient response characteristics are poor[2-4]. When the primary side voltage changes suddenly, and the secondary side output voltage cannot immediately respond to the change of the primary voltage.

In order to realize the wide-band measurement of CVT[5], which is widely used at present, the CVT equipment running on the spot is slightly modified, and a large capacitor $\mathrm{C} 3$ is connected in series to form a hybrid CVT, which realizes the measurement of transient overvoltage, and have the characteristics of convenient installation, stable and reliable operation, wide frequency coverage and fast response speed. This paper draws on relevant research results, combined with the actual parameter values of the series-parallel CVT of $110 \mathrm{kV}$ voltage level, built a high-frequency simulation model of the series-parallel CVT in ATP-EMTP, and the partial pressure characteristics of the capacitor C3 under different conditions are analyzed and verified by experiments.

\section{Hybrid CVT structure and principle}

Hybrid CVT is a series connection of low-voltage capacitor $\mathrm{C} 3$ between the low-voltage terminal of the medium-voltage capacitor $\mathrm{C} 2$ of the traditional capacitive voltage transformer and the ground, and it has the original measurement function of the CVT and the transient voltage measurement function at the same time. The basic structure principle of the Hybrid CVT is shown in Figure 1, which consists of two parts: a capacitor voltage divider unit and an electromagnetic unit.

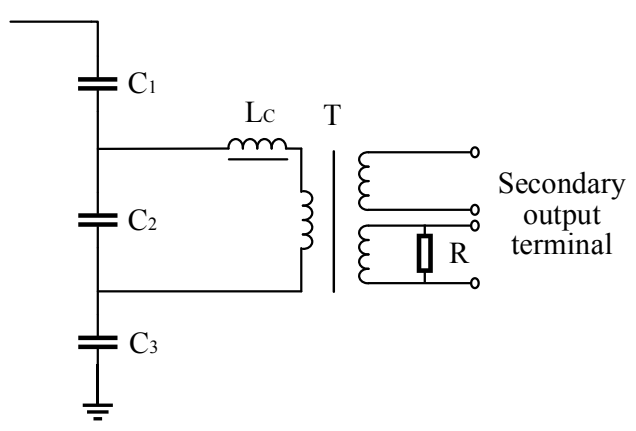

Figure 1. Hybrid CVT structure diagram

\footnotetext{
1 e-mail: nieyu2885@outlook.com
} 
The capacitor voltage divider unit includes the upper section high voltage capacitor $\mathrm{C} 1$, the lower section voltage divider capacitor $\mathrm{C} 2$, and the series connected low voltage capacitor $\mathrm{C} 3$. The function of the capacitor voltage divider unit is to extract the high voltage U1 on the primary side into the output voltage UC2 of the lower capacitor. The relationship between the output voltage UC2 of the traditional CVT capacitor C2 and the primary side voltage can be expressed as

$$
\frac{U_{1}}{U_{C_{2}}}=\frac{C_{1}+C_{2}}{C_{1}}
$$

Then the voltage divider ratio $\mathrm{KC}$ of the capacitor voltage transformer can be expressed as

$$
K_{\mathrm{C}}=\frac{C_{1}+C_{2}}{C_{1}}
$$

Since the capacitance value of $\mathrm{C} 3$ connected in series is much larger than $\mathrm{C} 1$ and $\mathrm{C} 2$, the relationship between the output voltage of capacitor $\mathrm{C} 2$ and the primary side voltage can be expressed as

$$
\frac{U_{1}}{U_{C_{2}}^{\prime}}=\frac{C_{1}+C_{2}}{C_{1}}+\frac{C_{2}}{C_{3}} \approx \frac{C_{1}+C_{2}}{C_{1}}
$$

Therefore, the voltage division ratio of the original CVT remains basically unchanged, $\mathrm{C} 3$ has no effect on

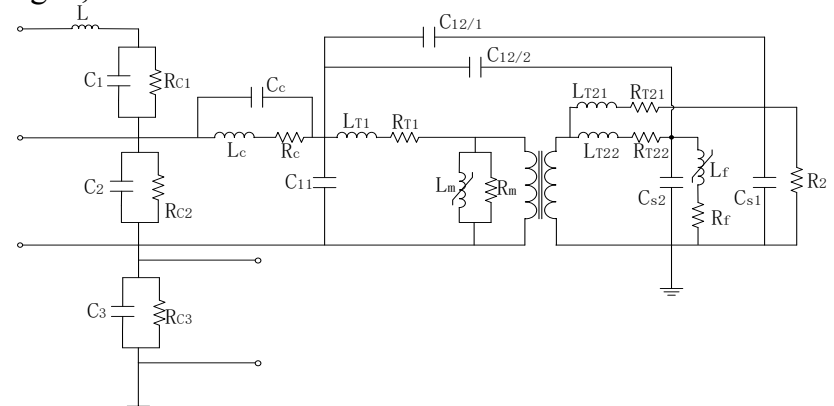

Figure 2. High-frequency transient simulation model of hybrid CVT

Capacitors C1, C2, C3 (including its dielectric loss $\mathrm{RC} 1, \mathrm{RC} 2, \mathrm{RC} 3$ and lead parasitic inductance $\mathrm{L}$ ); Compensation reactor LC (including its resistance RC and stray capacitance $\mathrm{CC}$ ); intermediate transformer (RT1 and LT1 are the resistance and inductance of the primary winding of the intermediate transformer, $\mathrm{Rm}$ and $\mathrm{Lm}$ are the resistance and inductance of the excitation branch of the intermediate transformer, RT21, RT22, LT21 and LT22 are the resistance and inductance of the load and damping winding on the secondary side of the intermediate transformer; the equivalent inductance Lf and equivalent resistance $\mathrm{Rf}$ of the fast saturation reactor; the voltage output of the secondary terminal of the CVT electromagnetic unit.

The electromagnetic unit includes a compensation reactor $\mathrm{LC}$, a medium voltage transformer $\mathrm{T}$, a damper $\mathrm{R}$ and a secondary output terminal. The electromagnetic unit connects the extracted lower capacitor voltage UC2 to the primary winding of the medium-voltage transformer, and transforms it into the secondary voltage signal used by the system metering, relay protection device, and control system through the medium-voltage transformer.

\section{Simulation Analysis of Hybrid CVT}

Combining the wave process theory of the voltage transformer for the establishment of the stray and distributed capacitance of the CVT model, as well as the actual measurement and calculation of the parameters of each component, the establishment of the corresponding high-frequency transient simulation model is shown in Figure 2. The voltage across $\mathrm{C} 3$ and the output voltage on the secondary side have been converted to the high voltage side according to the corresponding voltage division ratio and rated transformation ratio.

Side winding load R2; C12 is the stray capacitance between the primary and secondary windings; C11 is the stray capacitance of the primary winding; Cs1 and Cs2 are the stray capacitances of the secondary winding.

\subsection{Comparative analysis of the secondary side output voltage and the voltage across $\mathrm{C} 3$}

When the input voltage is $20 \mathrm{kV}$ lightning overvoltage, the waveforms of the input voltage, the secondary side output voltage and the voltage across $\mathrm{C} 3$ are shown in Figure 3.

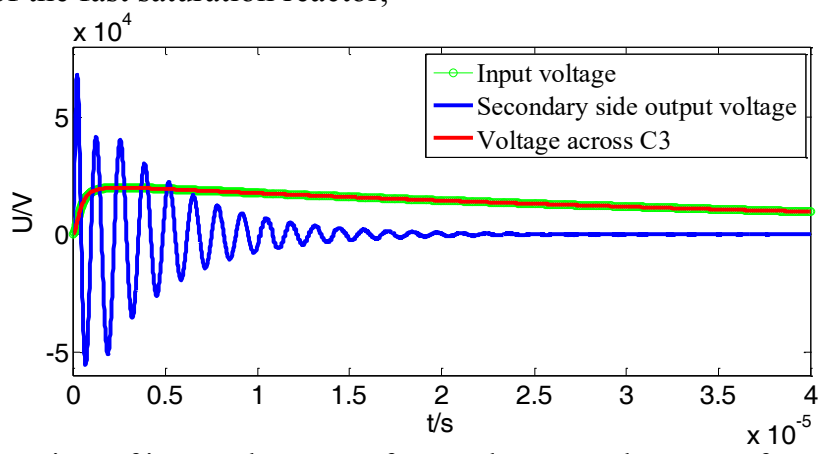

Figure 3. Comparison of input voltage waveform and output voltage waveform of each terminal 
It can be seen from the voltage waveform on the secondary side has a certain distortion, which cannot fully reflect the true waveform of the lightning overvoltage. From the above analysis, it is actually the electromagnetic unit structure that affects its existing direct measurement of lightning intrusion wave overvoltage waveform on the secondary side. The voltage waveform at both ends of $\mathrm{C} 3$ can better reflect the input voltage of the primary side.

\subsection{Simulation results and analysis of lightning impulse overvoltage}

Use ATP-EMTP to simulate the hybrid CVT model, input $+20 \mathrm{kV}$ lightning impulse voltage, the input voltage of the hybrid CVT and the waveform of the voltage across $\mathrm{C} 3$, different conditions The simulation results are as shown in the following figures.

(1) When there is no electromagnetic unit

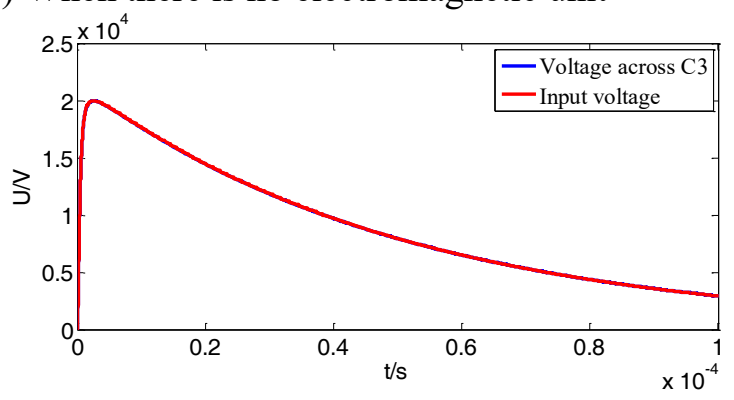

Figure 4. Output voltage and input voltage at both ends of $\mathrm{C} 3$ without electromagnetic unit
(2) When the electromagnetic unit is included and the CVT is running at no load

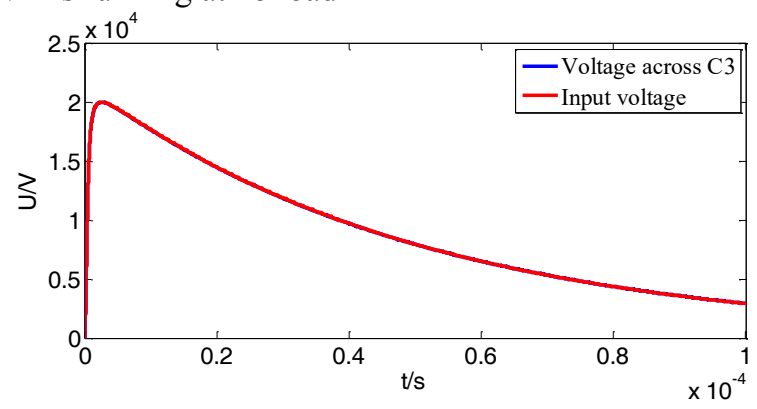

Figure 5. Output voltage and input voltage at both ends of electromagnetic unit $\mathrm{C} 3$ at no load

(3) Including electromagnetic unit, when 1a-1n winding is connected to $10 \mathrm{VA}$ rated load

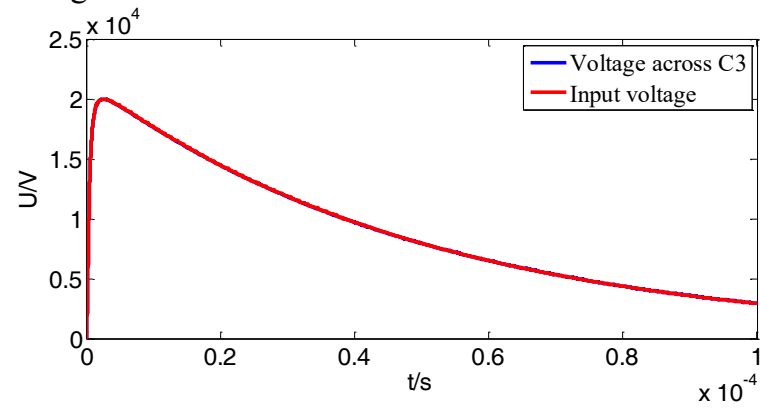

Figure 6. Output voltage and input voltage at both ends of electromagnetic unit $\mathrm{C} 3$ with $10 \mathrm{VA}$ load

Table 1. Comparison of parameter values

\begin{tabular}{ccccc}
\hline \multirow{2}{*}{ parameter } & $\begin{array}{c}\text { Input } \\
\text { voltage }\end{array}$ & $\begin{array}{c}\text { No electromagnetic } \\
\text { unit }\end{array}$ & $\begin{array}{c}\text { With electromagnetic unit } \\
\text { (1a-1n no load })\end{array}$ & $\begin{array}{c}\text { With electromagnetic unit(1a-1n } \\
\text { with 10VA load) }\end{array}$ \\
\hline $\begin{array}{c}\text { Wave head } \\
\text { time }(\mu \mathrm{s})\end{array}$ & 2.6 & 2.61 & 2.55 & 2.64 \\
$\begin{array}{c}\text { Half peak } \\
\text { time( } \mu \mathrm{s})\end{array}$ & 38.57 & 38.55 & 38.58 & 38.56 \\
Peak $(\mathrm{kV})$ & 20 & 6.4159 & 6.4068 & 6.4075 \\
$\begin{array}{c}\text { Partial pressure } \\
\text { ratio }\end{array}$ & 3117.5600 & 3117.2556 & 3121.6832 & 3121.3422 \\
\hline
\end{tabular}

The waveform parameter comparison of the simulation results is given in the table. It can be seen that the voltage divider ratios of the voltage output from both ends of C3 under different conditions are 3117.2556, 3121.6832 , and 3121.3422 , which are similar to the rated voltage divider ratio of the CVT, with the largest relative deviation. It is $0.13 \%$; the voltage output from both ends of $\mathrm{C} 3$ is similar to the impact time parameter of the input lightning overvoltage. The simulation results show that the two ends of the capacitor $\mathrm{C} 3$ connected in series in the hybrid CVT can be used to measure the lightning overvoltage waveform.

\section{Overvoltage waveform measurement test and analysis}

This article is designed to test the transient overvoltage of the $110 \mathrm{kV}$ hybrid CVT used in the test. The overvoltage test device used is the product used in the test produced by Taikai Transformer Factory, the model is TYD110/ $\sqrt{3}-0.01 \mathrm{H}$-hybrid Type CVT. The specific wiring diagram of the whole set of device test is shown in Figure 7. The parameters of this $110 \mathrm{kV}$ hybrid CVT are shown in Table 2. The capacitances of $\mathrm{C} 1, \mathrm{C} 2$, and $\mathrm{C} 3$ are $10200 \mathrm{pF}, 64530 \mathrm{pF}$, and $27.45 \mu \mathrm{F}$, respectively. The voltage source of the impulse voltage test is a $20 \mathrm{kV}$ impulse voltage generator SUG255TX, and the sampling 
frequency of the test oscilloscope is $5 \mathrm{GHz}$. Select the three channels in the oscilloscope to sequentially connect the lightning impulse voltage signal output by the attenuation probe; the voltage signal output from the secondary side terminals $1 \mathrm{a}-1 \mathrm{n}$ of the intermediate transformer of the electromagnetic unit through the coaxial shielded cable; the two ends of $\mathrm{C} 3$ are output through the coaxial shielded cable Voltage signal.

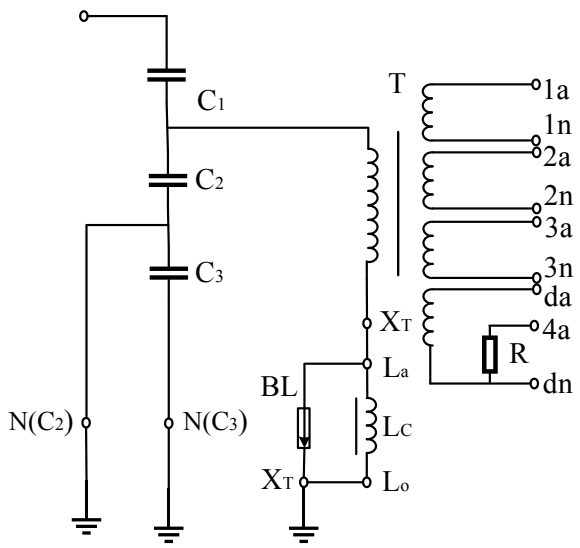

Figure 7. Test wiring diagram

Table 2. Main parameters of $110 \mathrm{kV}$ hybrid CVT for test

\begin{tabular}{ccccc}
\hline Parameter name & Parameter value \\
\hline Rated primary voltage $(\mathrm{kV})$ & \multicolumn{4}{c}{$110 / \sqrt{3}$} \\
Medium voltage transformer rated voltage $(\mathrm{kV})$ & $1 \mathrm{a}-1 \mathrm{n}$ & $2 \mathrm{a}-2 \mathrm{n}$ & $3 \mathrm{a}-3 \mathrm{n}$ & $\mathrm{da}-\mathrm{dn}$ \\
Secondary winding terminal mark & $0.1 / \sqrt{3}$ & $0.1 / \sqrt{3}$ & $0.1 / \sqrt{3}$ & 0.1 \\
Rated secondary voltage $(\mathrm{kV})$ & 0.2 & 0.5 & $3 \mathrm{P}$ & $3 \mathrm{P}$ \\
Accuracy & 10 & 10 & 10 & 10 \\
Rated output(VA) &
\end{tabular}

waveform comparison between the input voltage and the voltage across $\mathrm{C} 3$ is shown in the figure below.

\subsection{Power frequency voltage test results}

The power frequency voltage generator inputs $1200 \mathrm{~V}$ power frequency sinusoidal alternating current, and the

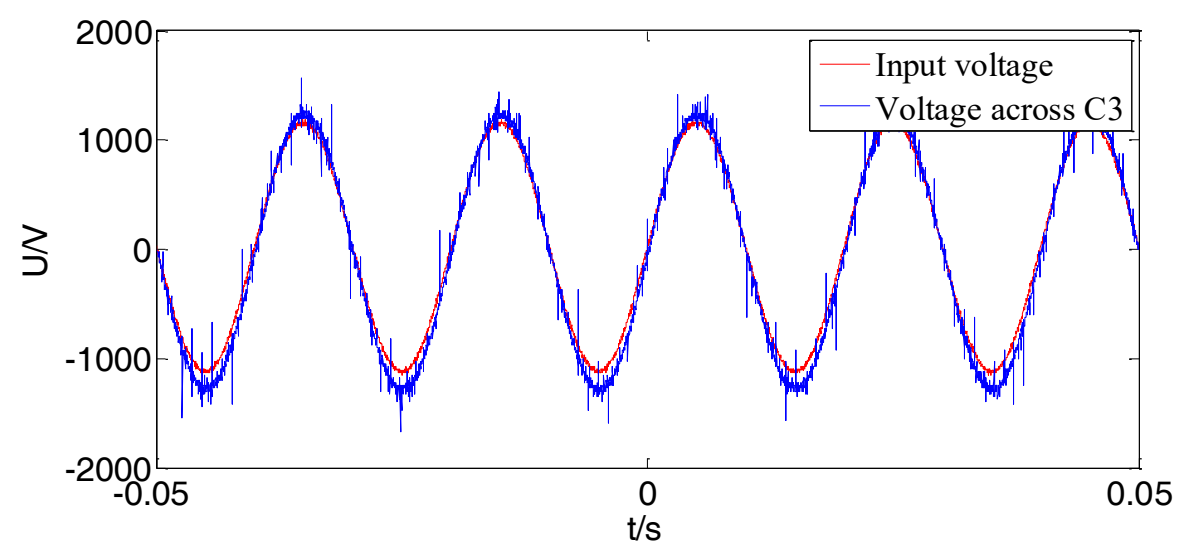

Figure 8. The input voltage and the voltage waveform at both ends of $\mathrm{C} 3$

The voltage waveform obtained through both ends of C3 is basically the same as the input voltage waveform. There is almost no deviation in frequency, and there is only a difference in amplitude. From the voltage waveform obtained from multiple measurements, the voltage divider ratio can be calculated: the voltage divider at both ends of $\mathrm{C} 3$ The ratio is similar to the calculated rated partial pressure ratio, and the relative deviation is about $3 \%$.

\subsection{Impulse voltage test results}

When the impulse generator inputs a $20 \mathrm{kV}$ lightning overvoltage, the waveforms of the input voltage and the secondary side output voltage are shown in the figure below. 


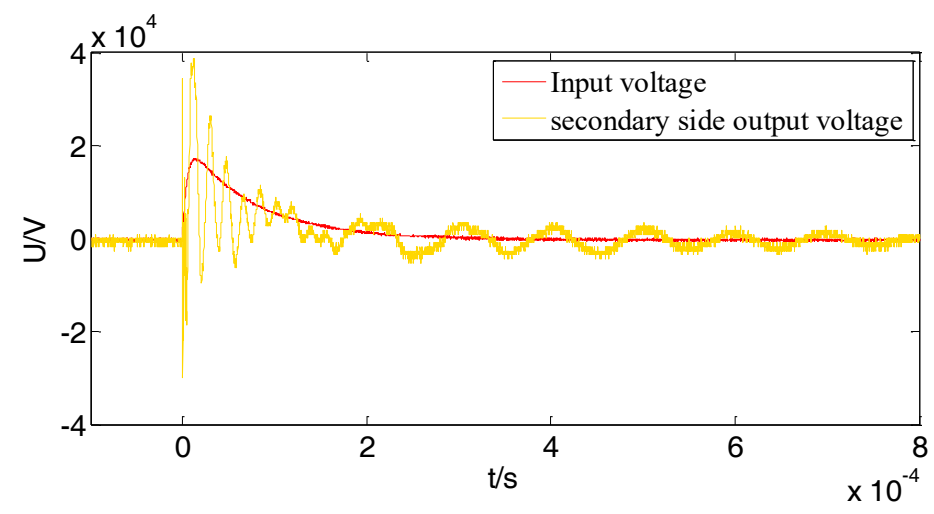

Figure 9. Input voltage and secondary side output voltage waveform

When the input amplitude is $+5 \mathrm{kV}$ lightning overvoltage, the waveforms of the input voltage and the voltage across $\mathrm{C} 3$ are shown in the figure below.

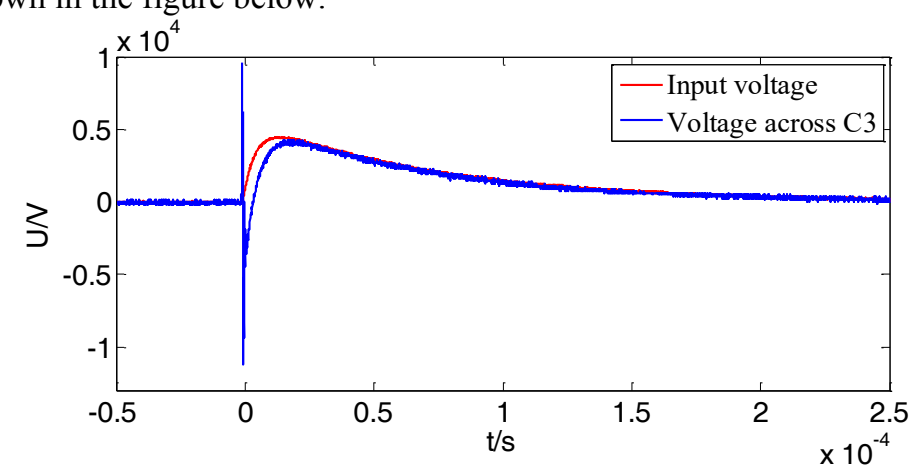

Figure 10. The input voltage and the voltage waveform at both ends of $\mathrm{C} 3$

\section{Conclusion}

This paper studies the characteristics of C3 partial pressure in a series-parallel CVT, uses ATP-EMTP software to simulate it, and conducts a power frequency voltage test and an impulse voltage test respectively, and analyzes the voltage waveform output at both ends of $\mathrm{C} 3$, including Voltage ratio, voltage amplitude, frequency deviation and impact time parameters. The simulation analysis results show that the measurement of transient overvoltage can be realized by the way of C3 partial pressure. The result of the test shows that when a lightning overvoltage is applied to the hybrid CVT, and it cannot be accurately transmitted by the secondary side. The voltage waveform at both ends of C3 does not oscillate significantly. However, the occurrence of similar shock phenomenon, considering the factors that may be derived from the measurement method, such as the influence of the matching impedance of the oscilloscope on the measurement results, further improvements and tests are needed. The above results are still of practical value for designing actual products and optimizing product performance.

\section{Acknowledgments}

This article is one of the phased achievements of Hebei Province's key research and development project" Broadband and Large Dynamic Range Current and
Voltage Measurement Technology Research and Equipment Development for Clean Energy"; The item number is 19212103D; The project leader is Guo Zhizhong; Start and end time 2019.05-2021.12.

\section{References}

1. Yang Jian, Cheng Hanxiang, Yu Yin, Li Lei. Current status and development of voltage transformers in power systems[J]. Electrical Engineering, 2015(07): 6-9+23.

2. Wang Liming, Fang Bin. Simulation of transient characteristics of $500 \mathrm{kV}$ capacitive voltage transformer[J]. High Voltage Technology, 2012, 38(09):2389-2396.

3. Yang Gang, Ran Zifeng, Arts, Yan Zheng, Li Jianming. Verification of Transient Characteristics of Capacitor Voltage Transformer[J].Sichuan Electric Power Technology,2013,36(06):87-91.

4. Xie Zenan, Zhao Wenbin, Zhao Dandan. Improved transient overvoltage monitoring and transient component extraction method[J]. Water Power Energy Science,2020,38(06):196-200.

5. Wen Yi, Chen Shaoqing. Research on CVT transient overvoltage response [J]. Power Capacitor and Reactive Power Compensation, 2014, 35(01): 45$50+59$. 